\title{
Pregnancy after bariatric surgery - a narrative literature review
}

\author{
Anna Różańska-Walędziak ${ }^{1}$, Paweł Bartnik ${ }^{1}$, Joanna Kacperczyk-Bartnik ${ }^{1}$, Krzysztof Czajkowski ${ }^{1}$, Maciej Walędziak², \\ Andrzej Kwiatkowski² \\ ${ }^{1} 2^{\text {nd }}$ Department of Obstetrics and Gynecology, Medical University of Warsaw, Warsaw, Poland \\ ${ }^{2}$ Department of General, Oncological, Metabolic and Thoracic Surgery, Military Institute of Medicine, Warsaw, Poland
}

Videosurgery Miniinv 2021; 16 (1): 30-37

DOI: https://doi.org/10.5114/wiitm.2020.99281

\begin{abstract}
The purpose of this review was to analyze the literature about pregnancy after bariatric surgery. We searched for available articles on the subject from the last decade (2010 to 2020). The positive impact of bariatric surgery on the level of comorbidities and pregnancy and neonatal outcomes cannot be overrated. Weight loss after bariatric surgery reduces the incidence of obesity-related conditions in pregnancy. A pregnancy in a woman after bariatric surgery should be considered a high-risk pregnancy and taken care of by a multidisciplinary team with appropriate micronutrient and vitamin supplementation provided. Optimum time to conception should be chosen following the international recommendations. Every woman after bariatric surgery should be aware of symptoms of surgical complications and immediately contact their surgeon in case of abdominal pain.
\end{abstract}

Key words: pregnancy, bariatric surgery, weight loss, intrauterine fetal growth retardation, gestational diabetes mellitus.

\section{Introduction}

Obesity has become a global healthcare problem, with $13 \%$ of the world population (650 million) estimated to be obese in 2016 [1]. Bariatric surgery (BS) is known to be the most effective method of treatment, with the most durable weight loss and the greatest reduction of concomitant diseases [2, 3]. Women constitute the majority of patients undergoing bariatric treatment, most of them of reproductive age. Obesity is associated with many comorbidities, e.g. hypertension, diabetes mellitus and obstructive sleep apnea, but also influences fertility, the course of pregnancy and neonatal outcomes [4]. Obesity in pregnancy increases the risk of gestational diabetes mellitus (GDM), pregnancy-induced hypertension $(\mathrm{PIH})$, prolonged labor, vacuum delivery, cesarean section, congenital anomalies and large for gestational age (LGA) infants [5]. Bariatric surgery reduces the risk of GDM, PIH, LGA, but also increases the risk of intrauterine growth retardation of the fetus (IUGR) and the proportion of small for gestational age (SGA) infants [6]. The alteration of the gastrointestinal absorption, hormone and metabolic changes may affect maternal and fetal well-being. Among the confirmed factors in the pathogenesis of impaired fetal growth are maternal micronutrient and vitamin deficiencies, but there are also other factors involved. The influence of BS on pregnancy and neonatal outcomes is subject to numerous studies. Pregnancy is associated with changes in body weight so that the influence of pregnancy and gestational weight gain on BS long-term outcomes remains an important question.

\section{Aim}

The aim of our review was to present recent studies about the relations between BS and pregnancy.

\section{Address for correspondence}

Maciej Walędziak MD, PhD, Department of General, Oncological, Metabolic and Thoracic Surgery, Military Institute of Medicine, Warsaw, Poland, e-mail: maciej.waledziak@gmail.com 
We divided our review into sections presenting the problems we considered the most important: the influence of pregnancy on weight loss after BS, the incidence of surgical complications in pregnancy, the importance of time to conception interval, micronutrient and vitamin deficiencies, fetal growth impairment, pregnancy-induced hypertension, gestational diabetes mellitus and contraception.

\section{Influence of pregnancy on weight loss after bariatric surgery}

Most studies about BS and pregnancy are focused on the impact of BS on pregnancy and neonatal outcomes. The question about the influence of pregnancy on long-term results of BS is of utmost importance both for patients and bariatric surgeons. There is evidence that pregnancy does not have negative effects on the weight loss after BS. Weight loss after 5 years from the operation is comparable between patients who became pregnant after the operation and those who were not. The neutral effect of pregnancy on BS outcomes was confirmed in a recent study by Brönnimann et al., who compared the excess body mass index (BMI) loss after 5-year follow-up between women with and without a history of pregnancy and found it to be similar in both groups [7]. Quyên Pham et al. analyzed the history of weight loss of 84 women who became pregnant after BS and concluded that pregnancy after BS slowed down the pace of weight loss, but eventually did not affect weight loss after 5-year follow-up when compared to the control group of women without a history of pregnancy after BS [8]. Rottenstreich et al. conducted a cross-sectional case-control study that included 80 women who became pregnant after laparoscopic sleeve gastrectomy (LSG) matched with 80 controls for preoperative BMI, age and follow-up duration. After a follow-up of more than 5 years, they found no differences in long-term weight loss results [9]. Alatishe et al. conducted a study in a group of women after BS and did not find any differences in \%EWL between those who became pregnant and those who did not [10]. Nevertheless, there are studies contradicting these results. Froylich et al. matched 62 patients after BS who were pregnant and had a delivery (before or after BS) with a control cohort of 92 patients after BS who had never conceived and found excess weight loss (\%EWL) of $68.0 \%$ in the delivery group vs $53.0 \%$ in the group of subjects who had never conceived. They concluded that a pregnancy before BS resulted in reduced weight loss after BS [11].

\section{Surgical complications during pregnancy}

The most common complications after BS in pregnancy are internal herniation following RYGB and gastric band slippage following adjustable gastric banding (AGB) [5].

The incidence of internal herniation in pregnancies after a Roux-en-Y gastric bypass (RYGB) was reported to be about $8 \%$ [12]. The most frequent symptoms of internal herniation are upper abdominal pain, nausea and vomiting, which can easily be mistaken for early pregnancy symptoms [13]. Severe abdominal pain during pregnancy can increase the risk of uterine contractions, preterm delivery and SGA infants [14]. Previous closure of mesenteric defects does not exclude the possibility of internal herniation in pregnancy. In a review of 22 cases, the most common location of the hernia was Petersen's space [15]. Women after RYGB should be advised not to delay a consultation with a bariatric specialist in case of symptoms suggesting internal herniation as there are reports suggesting a higher incidence of maternal and fetal death in case of intervention after more than $48 \mathrm{~h}$ from the symptoms onset [16, 17]. Any pregnant woman after RYGB presenting with abdominal pain should be assessed for the possibility of diagnosis of internal hernia [13].

The risk of gastric band slippage is increased in pregnancy due to vomiting and higher intraabdominal pressure. Some reports suggest incidence of slippage of $12 \%$ compared to $3-5 \%$ to the general population after $A G B[18,19]$. The symptoms of band slippage may also be mistaken for pregnancy symptoms [20].

\section{Time to conception}

International recommendations about pregnancy after BS (by Shawe et al. and the American College of Obstetricians and Gynecologists) agree that pregnancy should be postponed until the end of the rapid catabolic period of weight loss $[5,6]$. The suggested interval between the surgery and conception differs between recommendations and ranges from 12 to 24 months. Pregnancies started before the end of the rapid catabolic period are at higher risk of miscarriage, fetal malnutrition and impaired growth 
Table I. Recommended daily dosage of micronutrients and vitamins for (pre)pregnancy supplementation (after Shawe J et al. Pregnancy after bariatric surgery: consensus recommendations for periconception, antenatal and postnatal care. Obes Rev 2019; 20: 1507-22)

\begin{tabular}{|c|c|}
\hline Micronutrient & Recommended daily dosage \\
\hline Folic acid & $0.4 \mathrm{mg}$ (4-5 mg if obese or diabetic) \\
\hline Calcium & 1200-1500 mg (including dietary intake) \\
\hline Vitamin D & $>40 \mu \mathrm{g}(1000 \mathrm{IU})$ \\
\hline Iron & 45-60 mg (elemental iron) \\
\hline Copper & $2 \mathrm{mg}$ \\
\hline Zinc & 8-15 mg per 1 mg copper \\
\hline Thiamine & $>12 \mathrm{mg}$ \\
\hline Vitamin K & $90-120 \mu g$ \\
\hline Vitamin E & $15 \mathrm{mg}$ \\
\hline Vitamin A & 5000 IU (as B carotene) \\
\hline Selenium & $50 \mu g$ \\
\hline
\end{tabular}

$[5,6,21]$. Some authors emphasize the need of a patient-centered approach in assessing the optimum time for conception. Mahawar et al. suggested that instead of imposing a fixed time interval after the operation, it would be better to advise conceiving after at least 2 months of stable weight after the weight loss period [22]. Some studies suggest that a time to birth interval of less than 2 years was associated with a higher risk of preterm delivery, neonatal intensive care unit (NICU) admission and SGA infants [23].

Contrary to the international recommendations about the optimum time for pregnancy after bariatric surgery, there are studies showing no differences between pregnancy and neonatal outcomes if the time of conception followed the recommended time interval between BS and pregnancy. The analyzed endpoints were: preterm deliveries, birth weight, SGA neonates, NICU admissions, gestational weight gain (GWG), hyperemesis, nutritional deficiencies, GDM and PIH [24-27].

\section{Pregnancy and nutritional deficiencies after bariatric surgery}

BS, especially malabsorptive procedures, leads to various micronutrient and vitamin deficiencies due to changes in gastric $\mathrm{pH}$, dumping syndrome and absorption changes [28]. There are international guidelines indicating the optimum dietary supplementation after BS and during pregnancy after BS; however, the problem remains in patients' adherence to the recommendations, which decreases with increasing time since surgery $[5,6]$. The level of deficiencies rises with the decrease of patients' adherence to the recommendations. Maternal micronutrient and vitamin deficiencies may lead to impaired fetal growth [29,30].

The recommended minimum protein intake during pregnancy is $60 \mathrm{~g}$ of protein a day, although it should be adjusted for the patient's BMI and lean body mass. Rapidly absorbed carbohydrates should be avoided due to the risk of early and late postprandial syndrome. Pregnancy should be planned with the help of a multidisciplinary team and a multivitamin and mineral supplement should be taken prior to and throughout pregnancy [5]. Some patients restrict the daily caloric intake against medical advice because of their fear of regaining weight, sometimes to a level that may negatively affect the fetal well-being and intrauterine growth. The role of dietitian nutritionists and psychologists in the multidisciplinary care of a pregnant woman after BS cannot be overvalued. The suggested daily supplementation in pregnancy is presented in Table I.

The most commonly diagnosed deficiency is maternal anemia, often diagnosed before pregnancy. The incidence and level of maternal anemia increases in pregnancy due to higher demand of the developing fetus [31-33]. The incidence of maternal anemia during pregnancy is higher after malabsorptive procedures [34, 35]. Coupaye et al. in a study including 123 pregnancies after BS found a positive correlation between the risk of SGA infants and maternal protein intake and a negative correlation with the maternal iron status [36].

Rottenstreich et al. conducted a systematic review of 27 studies on maternal nutritional deficiencies in pregnancies after BS. The deficiencies found after both restrictive and malabsorptive procedures were iron, folate, vitamin $B_{1}, B_{12}$ and D. Additionally, the researchers found that there was an increased risk of maternal anemia in positive correlation with the time to conception length [37]. The level of circulating vitamin $\mathrm{K}_{1}$ is lower in pregnant patients after BS and supplementation is of utmost importance to prevent fetal and neonatal intracranial hemorrhages [38]. 
Low blood glucose levels, often diagnosed after RYGB and other types of bypass BS, can lead to impairment of fetal growth. As stated by Rottenstreich et al., maternal hypoglycemia and subsequent fetal hypoglycemia are a common consequence of BS, especially malabsorptive surgery (MS). After the oral glucose tolerance test (OGTT), hypoglycemia was found in more than $50 \%$ of patients after BS and $83 \%$ of patients after MS [39]. Low inflow of glucose to the fetus may result in IUGR and SGA infants and maternal hypoglycemia may be present for a substantial portion of time in mothers after MS. OGTT is currently considered an unacceptable method of screening for GDM in women after all types of BS procedures except for AGB, which does not cause direct metabolic changes. Late postprandial syndrome and reactive hypoglycemia occurring after BS lead to lessened tolerance and accuracy of OGTT, excluding it as a method of diagnosing GDM in pregnant patients after BS. OGTT should be substituted with monitoring of capillary blood glucose levels between the $24^{\text {th }}$ and $28^{\text {th }}$ week of gestation or continuous glucose monitoring [5, 40-42].

\section{Fetal growth impairment}

The vast majority of studies show a decreased rate of GDM, PIH and LGA in pregnancies after BS and an increased risk of IUGR and SGA infants.

There is an important question whether all types of bariatric procedures lead to similar pregnancy and neonatal outcomes, or whether there are differences. LSG remains the most popular BS procedure in the world and its influence on pregnancy course has to be well established. The risk of SGA infants after MS has been confirmed in many studies [43]. One of the most important studies in the field was a national Swedish cohort study, which included 670 pregnancies after BS, 98\% out of whom had a history of RYGB. The study presented a more than two-fold increase in risk of SGA neonates after BS (15.6 vs. 7.6\%) [44]. Kjaer et al. presented an analysis of 339 pregnancies after BS; $84.4 \%$ after RYGB. The risk of SGA infants was 2.3 times higher after BS than in the control group [45]. An increase in the risk of SGA neonates after RYGB was also found by Belcastro et al. [46].

There are also studies comparing purely restrictive procedures, such as laparoscopic adjustable gastric banding (LAGB) with MS. Chevrot et al. found a two-fold increase of the risk of SGA infants after RYGB, compared to LAGB and in the general population [47]. In a study by Facchiano et al. the mean birth weight of neonates was lower after RYGB than LAGB [48]. A meta-analysis of 33 studies by Akhter et al. did not find any correlation between incidence of SGA infants and restrictive surgery (RS), contrary to an increased risk after MS [43]. A study by Aricha-Tamir et al., who analyzed paired pregnancies before and after BS, found no association between BS and the risk of SGA infants [49].

Some studies present a comparable risk of IUGR and SGA neonates after RS and MS [50]. Coupaye et al. found in their study, in which they included 123 pregnancies after BS (77 after RYGB and 46 after SG) a comparable rate of IUGR and SGA infants between both types of procedures [36]. No differences in the risk of SGA neonates between pregnancies after RS and BS were also found by Sheiner et al. and the risk of IUGR was 2.5 times higher after BS compared to the control group [51]. The importance of the risk of SGA infants after LSG was analyzed by Rottenstreich et al. in a recent case-control study that included 119 pregnancies after LSG compared to obese controls. They found a more than three-fold increase in the incidence of SGA infants after LSG (4.3 vs. 14.3\%), having confirmed an increased risk of SGA infants after RS [52]. These findings suggest that there are more mechanisms involved in the pathogenesis of growth restriction after $\mathrm{BS}$, not only the absorption changes.

\section{Pregnancy-induced hypertension and pre-eclampsia}

Pregnancy-induced hypertension is defined as de novo onset of hypertension diagnosed after the $20^{\text {th }}$ week of gestation, with $>140 \mathrm{~mm} \mathrm{Hg}$ systolic or $>90 \mathrm{~mm} \mathrm{Hg}$ diastolic. Preeclampsia is a multi-systemic disease with at least one new-onset condition complicating the course of $\mathrm{PIH}$, including proteinuria and other maternal organ dysfunction: renal insufficiency, liver involvement, neurological and hematological complications. Both $\mathrm{PIH}$ and preeclampsia can negatively affect the pregnancy course, leading to adverse pregnancy and neonatal outcomes, including preterm delivery and IUGR. Preeclampsia remains the leading cause of maternal and neonatal morbidity and mortality worldwide [53]. The pathogenesis of preeclampsia starts with abnormalities in 
placenta development, angiogenesis alterations and abnormal trophoblastic invasion [54, 55]. Obesity-related metabolic factors may influence the cytotrophoblast and endothelial dysfunction, increasing the risk of preeclampsia [56]. Obesity is a major risk factor of developing preeclampsia, with a strong positive association between pre-pregnancy BMI and the risk of preeclampsia, doubling with each $5-7 \mathrm{~kg} / \mathrm{m}^{2}$ increase in pre-pregnancy BMI [57]. The risk of preeclampsia is three-fold higher in obese women compared to the general population [31]. Reducing GWG in obese patients decreases the risk of preeclampsia, but increases the incidence of SGA infants [58]. Most studies show that BS is associated with a significant reduction of risk of preeclampsia. A meta-analysis by Galazis et al. based on 17 cohort and case-control studies showed a lower incidence of preeclampsia after BS with an OR of 0.45 [59]. Bennett et al. analyzed 269 pregnancies before and 316 after BS and found a substantial reduction of incidence of preeclampsia $(O R=0.20)$ and PIH $(O R=0.16)[60]$. The reduction of incidence of $\mathrm{PIH}$ and preeclampsia in patients after BS has been confirmed in numerous studies and meta-analyses [30, 31, 61-65].

\section{Gestational diabetes mellitus}

Obesity is a well-known risk factor for developing diabetes mellitus, the incidence of which can be significantly reduced after bariatric surgery $[66,67]$. Gestational diabetes mellitus is a condition of abnormal maternal glucose tolerance that is diagnosed for the first time during pregnancy. High pre-pregnancy BMI is a risk factor for GDM and developing type 2 diabetes mellitus after pregnancy [68]. GDM can lead to disturbances in fetal development, including fetal macrosomia and a four to five times higher rate of congenital malformations than in the non-diabetic population [69]. BS and the following reduction of body weight leads to an important decrease in the rate of GDM, when compared to the obese population, and in some studies even to the general non-obese population. The vast majority of studies on the influence of BS on the pregnancy course confirm a significant reduction in the incidence of GDM after BS [30, 42, 70, 71]. Johansson et al. found a more than three-fold reduction of the incidence of GDM in patients after BS compared to the obese population, and the results were corroborated in an analysis by Burke et al. [44, 72]. In one of our studies we observed a twofold decrease in the proportion of GDM in patients after
BS compared to the general non-obese population [34]. A meta-analysis by Galazis et al. based on 17 studies also demonstrated a two-fold decrease in incidence of GDM [59]. The decrease in incidence of GDM has been confirmed in other studies $[19,49,73]$.

\section{Contraception}

Women after bariatric surgery are recommended to delay conception for the period of rapid weight loss of at least 12 to 24 months, and pre-operative counseling about appropriate and effective methods of birth control is of utmost importance. Mengesha et al. observed in their study that even a single counseling visit ameliorated the rate of optimum contraception use after BS [74].

According to the consensus recommendations by Shawe et al., BS can impair the absorption of both estrogen and progestagen components of oral contraceptives and therefore combined oral contraception can be less effective after BS [5, 75]. However, studies confirming those statements are based on populations after older types of procedures, such as BPD, AGB or jejunoileal bypass [76-79]. There is no level 1 evidence confirming the compromised absorption of combined oral contraception, and some new studies suggest normal pharmacokinetics of etonogestrel after BS [80]. Further studies are necessary to evaluate the real risk of reduced efficacy of oral contraception after BS.

Additionally, oral contraception should be advised against in obese pre-operative patients and those still affected by obesity after BS due to increased risk of venous thromboembolism [81, 82]. Nevertheless, there are studies presenting the same proportion of oral contraception use before and after BS, sometimes even at the level of $15 \%$ of the population, which further emphasizes the importance of pre-operative counseling $[83,84]$.

Patients after BS should be advised to use long-acting reversible methods of contraception, such as intrauterine devices and progestagen implants, followed by non-hormonal barrier methods in women preferring those (male and female condoms may be suitable; diaphragms require adjustment of size after every $3 \mathrm{~kg}$ of weight change) [5, 85-88].

\section{Conclusions}

Bariatric surgery has a positive impact on pregnancy outcomes through reduction of obesity-re- 
lated comorbidities. The proportion of gestational diabetes and preeclampsia in patients after BS is significantly decreased. However, there are also negative effects of BS on the pregnancy, such as a higher risk of IUGR and SGA infants, as well as maternal micronutrient and vitamin deficiencies. There is also a higher risk of surgical complications after BS, especially internal herniation after RYGB. A pregnancy in a woman after bariatric surgery should be considered a high-risk pregnancy, and a multidisciplinary team, including an experienced obstetrician, a bariatric surgeon, a dietitian nutritionist and a psychologist, should take care of every pregnant woman after weight loss surgery. Appropriate micronutrient and vitamin supplementation in accordance with current recommendations should be provided. An alternative form of screening for gestational diabetes mellitus has to be implemented. Optimum time to conception should be chosen following the guidelines, but also individually consulted in each case. Appropriate and efficient contraception should be introduced and birth control counseling should always be included in pre-operative care. Every woman after bariatric surgery should be aware of the symptoms of surgical complications and immediately contact their surgeon in case of abdominal pain.

\section{Conflict of interest}

The authors declare no conflict of interest.

\section{References}

1. Neylan CJ, Kannan U, Dempsey DT, et al. The surgical management of obesity. Gastroenterol Clin N Am 2016; 45: 689-703.

2. Lindekilde N, Gladstone BP, Lübeck M, et al. The impact of bariatric surgery on quality of life: a systematic review and meta-analysis. Obes Rev 2015; 16: 639-51.

3. Gloy VL, Briel M, Bhatt DL, et al. Bariatric surgery versus non-surgical treatment for obesity: a systematic review and meta-analysis of randomised controlled trials. BMJ 2013; 347: f5934.

4. Walędziak M, Różańska-Walędziak A, Kowalewski PK, et al. Present trends in bariatric surgery in Poland. Videosurgery Miniinv 2019; 14: 86-9.

5. Shawe J, Ceulemans D, Akhter Z, et al. Pregnancy after bariatric surgery: consensus recommendations for periconception, an tenatal and postnatal care. Obes Rev 2019; 20: 1507-22.

6. ACOG Practice Bulletin. Clinical Management Guidelines for Obstetrician-Gynecologists 2009; 113: 1405-13.

7. Brönnimann A, Jung MK, Niclauss N, et al. The impact of pregnancy on outcomes after bariatric surgery. Obes Surg 2020; 30: 3001-9.
8. Quyên Pham T, Pigeyre M, Caiazzo R, et al. Does pregnancy influence long-term results of bariatric surgery? Surg Obes Relat Dis 2015; 11: 1134-9.

9. Rottenstreich A, Shufanieh J, Kleinstern G, et al. The long-term effect of pregnancy on weight loss after sleeve gastrectomy. Surg Obes Relat Dis 2018; 14: 1594-9.

10. Alatishe A, Ammori BJ, New JP, Syed AA. Bariatric surgery in women of childbearing age. QJM 2013; 106: 717-20.

11. Froylich D, Corcelles R, Daigle CR, et al. The effect of pregnancy before and/or after bariatric surgery on weight loss. Surg Obes Relat Dis 2016; 12: 596-9.

12. Devlieger R, Jans G, Matthys C. Outcomes of pregnancy after bariatric surgery. N Engl J Med 2015; 372: 2266.

13. Wax JR, Pinette MG, Cartin A. Roux-en-Y gastric bypass-associated bowel obstruction complicating pregnancy-an obstetrician's map to the clinical minefield. Am J Obstet Gynecol 2013; 208: 265-71.

14. Petersen L, Lauenborg J, Svare J, Nilas L. The impact of upper abdominal pain during pregnancy following a gastric bypass. Obes Surg 2017; 27: 688-93.

15. Leal-González R, De la Garza-Ramos R, Guajardo-Pérez H, et al. Internal hernias in pregnant women with history of gastric bypass surgery: case series and review of literature. Int I Surg Case Rep 2013; 4: 44-7.

16. Vannevel V, Jans G, Bialecka M, et al. Internal herniation in pregnancy after gastric bypass: a systematic review. Obstet Gynecol 2016; 127: 1013-20.

17. Borghede MK, Vinter-Jensen L, Andersen JC, et al. Reconstruction of short bowel syndrome after internal hernia in a pregnant woman with previous bariatric surgery. Int I Surg Case Rep 2013; 4: 1100-3.

18. Carelli AM, Ren CJ, Youn HA, et al. Impact of laparoscopic adjustable gastric banding on pregnancy, maternal weight, and neonatal health. Obes Surg 2011; 21: 1552-8.

19. Chevrot A, Lesage N, Msika S, Mandelbrot L. Digestive surgical complications during pregnancy following bariatric surgery: experience of a center for perinatology and obesity. J Gynecol Obstet Biol Reprod 2016; 45: 372-9.

20. Haward RN, Brown WA, O'Brien PE. Does pregnancy increase the need for revisional surgery after laparoscopic adjustable gastric banding? Obes Surg 2011; 21: 1362-9.

21. Rottenstreich A, Elchalal U, Elazary R. Pregnancy after bariatric surgery: risks and benefits. Am J Obstet Gynecol 2018; 219: 310.

22. Mahawar KK, Graham Y, Small PK. Optimum time for pregnancy after bariatric surgery. Surg Obes Relat Dis 2016; 12: 1126-8.

23. Parent B, Martopullo I, Weiss NS, et al. Bariatric surgery in women of childbearing age, timing between an operation and birth, and associated perinatal complications. JAMA Surg 2017; 152: 128-35.

24. Yau PO, Parikh M, Saunders JK, et al. Pregnancy after bariatric surgery: the effect of time-to-conception on pregnancy outcomes. Surg Obes Relat Dis 2017; 13: 1899-905.

25. Dao T, Kuhn J, Ehmer D, et al. Pregnancy outcomes after gastric-bypass surgery. Am J Surg 2006; 192: 762-6.

26. Dolin CD, Chervenak J, Pivo S, et al. Association between time interval from bariatric surgery to pregnancy and maternal weight outcomes. J Matern Fetal Neonatal Med 2019; 1-7. doi: 10.1080/14767058.2019.1683156. 
27. Stentebjerg LL, Andersen LLT, Renault K, et al. Pregnancy and perinatal outcomes according to surgery to conception interval and gestational weight gain in women with previous gastric bypass. J Matern Fetal Neonatal Med 2017; 30: 1182-8.

28. Kominiarek MA. Preparing for and managing a pregnancy after bariatric surgery. Sem Perinatol 2011; 35: 356-61.

29. Slater C, Morris L, Ellison J, et al. Nutrition in pregnancy following bariatric surgery. Nutrients 2017; 9: 1338.

30. Guelinckx I, Devlieger R, Vansant G. Reproductive outcome after bariatric surgery: a critical review. Hum Reprod Update 2009; 15: 189-201.

31. Falcone V, Stopp T, Feichtinger $M$, et al. Pregnancy after bariatric surgery: a narrative literature review and discussion of impact on pregnancy management and outcome. BMC Pregnancy Childbirth 2018; 18: 507.

32. Nomura RMY, Dias MCG, Igai AMK, et al. Anemia during pregnancy after silastic ring Roux-en-Y gastric bypass: influence of time to conception. Obes Surg 2011; 21: 479-84.

33. Costa MM, Belo S, Souteiro P, et al. Pregnancy after bariatric surgery: maternal and fetal outcomes of 39 pregnancies and a literature review. J Obstet Gynaecol Res 2018; 44: 681-90.

34. Różańska-Walędziak A, Walędziak M, Bartnik P, et al. The influence of bariatric surgery on pregnancy and perinatal outcomes-a case-control study. J Clin Med 2020; 9: E1324.

35. Watanabe A, Seki Y, Haruta H, et al. Maternal impacts and perinatal outcomes after three types of bariatric surgery at a single institution. Arch Gynecol Obstet 2019; 300: 145-52.

36. Coupaye M, Legardeur H, Sami O, et al. Impact of Roux-en-Y gastric bypass and sleeve gastrectomy on fetal growth and relationship with maternal nutritional status. Surg Obes Relat Dis 2018; 14: 1488-94.

37. Rottenstreich A, Elazary R, Goldenshluger A, et al. Maternal nutritional status and related pregnancy outcomes following bariatric surgery: a systematic review. Surg Obes Relat Dis 2019; 15: $324-32$

38. Jans G, Guelinckx I, Voets W, et al. Vitamin K1 monitoring in pregnancies after bariatric surgery: a prospective cohort study. Surg Obes Relat Dis 2014; 10: 885-90.

39. Rottenstreich A, Elazary R, Ezra Y, et al. Hypoglycemia during oral glucose tolerance test among post-bariatric surgery pregnant patients: incidence and perinatal significance. Surg Obes Relat Dis 2018; 14: 347-53.

40. Adam S, Ammori B, Soran H. Pregnancy after bariatric surgery: screening for gestational diabetes. BMJ 2017; 356: j533.

41. Narayanan RP, Syed AA. Pregnancy following bariatric surgery medical complications and management. Obes Surg 2016; 26 : 2523-9.

42. Benhalima K, Minschart C, Ceulemans D, et al. Screening and management of gestational diabetes mellitus after bariatric surgery. Nutrients 2018; 10: 1479.

43. Akhter Z, Rankin J, Ceulemans D, et al. Pregnancy after bariatric surgery and adverse perinatal outcomes: a systematic review and meta-analysis. PLoS Med 2019; 16: e1002866.

44. Johansson K, Cnattingius S, Naslund I, et al. Outcomes of preg nancy after bariatric surgery. N Engl J Med 2015; 372: 814-24

45. Kjaer MM, Lauenborg J, Breum BM, Nilas L. The risk of adverse pregnancy outcome after bariatric surgery: a nationwide reg- ister-based matched cohort study. Am J Obstet Gynecol 2013; 208: 464.e1-5.

46. Belcastro MR, Neiger R, Ventolini G. Intrauterine growth restriction after bariatric surgery. J Neonatal Perinatal Med 2012; 4: 231-34.

47. Chevrot A, Kayem G, Coupaye M, et al. Impact of bariatric surgery on fetal growth restriction: experience of a perinatal and bariatric surgery center. Am J Obstet Gynecol 2016; 214: 655.e1-7.

48. Facchiano E, lannelli A, Santulli P, et al. Pregnancy after laparoscopic bariatric surgery: comparative study of adjustable gastic banding and Roux-en-Y gastric bypass. Surg Obes Relat Dis 2012; 8: 429-33.

49. Aricha-Tamir B, Weintraub AY, Levi I, Sheiner E. Downsizing pregnancy complications: a study of paired pregnancy outcomes before and after bariatric surgery. Surg Obes Relat Dis 2012; 8: 434-9.

50. Malik S, Teh JL, Lomanto D, et al. Maternal and fetal outcomes of Asian pregnancies after bariatric surgery. Surg Obes Relat Dis 2020; 16: 529-35.

51. Sheiner E, Balaban E, Dreiher J, et al. Pregnancy outcome in patients following different types of bariatric surgeries. Obes Surg 2009; 19: 1286-92.

52. Rottenstreich A, Elazary R, Levin G. Pregnancy after bariatric surgery and the risk of fetal growth restriction. Surg Obes Relat Dis 2018; 14: 1919-20.

53. Laganà AS, Giordano D, Loddo S, et al. Decreased endothelial progenitor cells (EPCS) and increased natural killer (NK) cells in peripheral blood as possible early markers of preeclampsia: a case-control analysis. Arch Gynecol Obstet 2017; 295 : 867-72.

54. Laganà AS, Vitale SG, Sapia F, et al. miRNA expression for early diagnosis of preeclampsia onset: hope or hype? J Matern Fetal Neonatal Med 2018; 31: 817-21.

55. Laganà AS, Favilli A, Triolo $O$, et al. Early serum markers of pre-eclampsia: are we stepping forward? J Matern Fetal Neonatal Med 2016; 29: 3019-23.

56. Spradley FT, Palei AC, Granger JP. Increased risk for the development of preeclampsia in obese pregnancies: weighing in on the mechanisms. Am J Physiol Regul Integr Comp Physiol 2015; 309: R1326-43.

57. Dixit A, Girling JC. Obesity and pregnancy. J Obstet Gynaecol 2008; 28: 14-23.

58. Wax JR. Risks and management of obesity in pregnancy: current controversies. Curr Opin Obstet Gynecol 2009; 21: 117-23.

59. Galazis N, Docheva N, Simillis C, et al. Maternal and neonatal outcomes in women undergoing bariatric surgery: a systematic review and meta-analysis. Eur J Obstet Gynecol Reprod Biol 2014; 181: 45-53.

60. Bennett WL, Gilson MM, Jamshidi R, et al. Impact of bariatric surgery on hypertensive disorders in pregnancy: retrospective analysis of insurance claims data. BMJ 2010; 340: c1662.

61. Yi X, Li Q, Zhang J, Wang Z. A meta-analysis of maternal and fetal outcomes of pregnancy after bariatric surgery. Int J Gynecol Obstet 2015; 130: 3-9.

62. Maggard MA, Yermilov I, Li Z, et al. Pregnancy and fertility following bariatric surgery: a systematic review. JAMA 2008; 300: 2286-96. 
63. Vrebosch L, Bel S, Vansant G, et al. Maternal and neonatal outcome after laparoscopic adjustable gastric banding: a systematic review. Obes Surg 2012; 22: 1568-79.

64. Magdaleno R, Pereira BG, Chaim EA, Turato ER. Pregnancy after bariatric surgery: a current view of maternal. obstetrical and perinatal challenges Arch Gynecol Obstet 2012; 285: 559-66.

65. Sheiner E, Willis K; Bariatric Surgery YY. Impact on pregnancy outcomes. Curr Diab Rep 2013; 13: 19-26.

66. Wojciak P, Pawłuszewicz P, Diemieszczyk I, et al. Laparoscopic sleeve gastrectomy: a study of efficiency in treatment of metabolic syndrome components, comorbidities and influence on certain biochemical markers. Videosurgery Miniinv 2020; 15: 136-47.

67. Sierżantowicz R, Ładny JR, Lewko J, Hady HR. Assessment of education effects on patient involvement and bariatric treatment outcome: an observational study. Videosurgery Miniinv 2020; 15: 157-65.

68. Corrado F, D’Anna R, Laganà AS, Di Benedetto A. Abnormal glucose tolerance later in life in women affected by glucose intolerance during pregnancy. J Obstet Gynaecol 2014; 34: 123-6.

69. Facchinetti F, Appetecchia M, Aragona C, et al. Experts' opinion on inositols in treating polycystic ovary syndrome and non-insulin dependent diabetes mellitus: a further help for human reproduction and beyond. Expert Opin Drug Metab Toxicol 2020; 16: 255-74.

70. Lesko J, Peaceman A. Pregnancy outcomes in women after bariatric surgery compared with obese and morbidly obese controls. Obstet Gynecol 2012; 119: 547-54.

71. Haseeb YA. A review of obstetrical outcomes and complications in pregnant women after bariatric surgery. Sultan Qaboos Univ Med I 2019; 19: e284-90.

72. Burke AE, Bennett WI, Janshidi RM, et al. Reduced incidence of gestational diabetes with bariatric surgery. J Am Coll Surg 2010; 211: 169-75.

73. Kwong W, Tomlinson G, Feig DS. Maternal and neonatal outcomes after bariatric surgery; a systematic review and meta-analysis: do the benefits outweigh the risks? Am J Obstet Gynecol 2018; 218: 573-80.

74. Mengesha BM, Carter JT, Dehlendorf CE, et al. Perioperative pregnancy interval, contraceptive counseling experiences, and contraceptive use in women undergoing bariatric surgery. Am J Obstet Gynecol 2018; 219: 81.e1-9.

75. Yska JP, van der Linde S, Tapper VV, et al. Influence of bariatric surgery on the use and pharmacokinetics of some major drug classes. Obes Surg 2013; 23: 819-25.

76. Gerrits EG, Ceulemans R, van Hee R, et al. Contraceptive treatment after biliopancreatic diversion needs consensus. Obes Surg 2003; 13: 378-82.

77. Weiss HG, Nehoda H, Labeck B, et al. Pregnancies after adjustable gastric banding. Obes Surg 2001; 11: 303-6.

78. Victor A, Odlind V, Kral JG. Oral contraceptive absorption and sex hormone binding globulins in obese women: effects of jejunoileal bypass. Gastroenterol Clin North Am 1987; 16: 483-91.

79. Andersen AN, Lebech PE, Sørensen TI, Borggaard B. Sex hormone levels and intestinal absorption of estradiol and D-norgestrel in women following bypass surgery for morbid obesity. Int J Obes 1982; 6: 91-6.
80. Ginstman C, Frisk J, Carlsson B, et al. Plasma concentrations of etonogestrel in women using oral desogestrel before and after Roux-en-Y gastric bypass surgery: a pharmacokinetic study. BJOG 2019; 126: 486-92.

81. Curtis KM, Tepper NK, Jatlaoui TC, et al. U.S. Medical Eligibility Criteria for Contraceptive Use, 2016. MMWR Recomm Rep 2016; 65: 1-103.

82. Butterworth J, Deguara J, Borg CM. Bariatric surgery, polycystic ovary syndrome, and infertility. J Obes 2016; 2016: 1871594. doi:10.1155/2016/187159424.

83. Różańska-Walędziak A, Bartnik P, Kacperczyk-Bartnik J, et al. The impact of bariatric surgery on menstrual abnormalities-a cross-sectional study. Obes Surg 2020; 10.1007/s11695020-04840-6.

84. Menke MN, King WC, White GE, et al. Contraception and conception after bariatric surgery. Obstet Gynecol 2017; 130: 979-87.

85. Hillman JB, Miller RJ, Inge TH. Menstrual concerns and intrauterine contraception among adolescent bariatric surgery patients. J Womens Health 2011; 20: 533-8.

86. Luyssen J, Jans G, Bogaerts A, et al. Contraception, menstruation, and sexuality after bariatric surgery: a prospective cohort study. Obes Surg 2018; 28: 1385-93.

87. Ciangura C, Corigliano N, Basdevant A, et al. Etonorgestrel concentrations in morbidly obese women following Roux-en-Y gastric bypass surgery: three case reports. Contraception 2011; 84: 649-51.

88. Graham Y, Wilkes S, Mansour D, Small PK. Contraceptive needs of women following bariatric surgery. J Fam Plann Reprod Health Care 2014; 40: 241-4.

Received: 28.06.2020, accepted: 10.08.2020. 